\title{
Diverse Effects of Different Akkermansia muciniphila Genotypes on Brown Adipose Tissue Inflammation and Whitening in a High-fat-diet Murine Model
}

\author{
Lulu Deng \\ Zhujiang Hospital \\ Zihao Ou \\ Zhujiang Hospital \\ Dongquan Huang \\ Zhujiang Hospital
}

Chong Li

Zhujiang Hospital

Zhi Lu

Zhujiang Hospital

Wanting Liu

Zhujiang Hospital

Feifan Wu

Zhujiang Hospital

Caihong Nong

Zhujiang Hospital

Jie Gao

Zhujiang Hospital

Yongzheng Peng ( $\nabla$ yzpeng1981@126.com )

Zhujiang Hospital

Research article

Keywords: Akkermansia muciniphila; genotype; brown adipose tissue; high-fat-diet

Posted Date: August 5th, 2019

DOl: https://doi.org/10.21203/rs.2.12409/v1

License: (c) (i) This work is licensed under a Creative Commons Attribution 4.0 International License.

Read Full License 
Version of Record: A version of this preprint was published at Microbial Pathogenesis on October 1st, 2020. See the published version at https://doi.org/10.1016/j.micpath.2020.104353. 


\section{Abstract}

Background The study aimed to investigate the differences of different Akkermansia muciniphila (A.muciniphila) genotypes on metabolic protective effects in mice with high-fat diet and explore possible mechanisms. Methods Male C57BL/ 6 mice were randomly divided into 6 groups, including high-fat diet (HFD)+ A.muciniphila I/II/PBS group, normal chow diet (NCD)+A.muciniphila I/ II /PBS group, respectively. Dietary intervention and A.muciniphila gavage were performed simultaneously. Blood glucose and lipid metabolism, brown adipose morphology and activities, and intestinal barrier function were examined after the mice were sacrificed. Results A.muciniphila gavage improved the impaired glucose tolerance, hyperlipidemia and liver steatosis in HFD mice, and that A.muciniphila II was not as effective as A.muciniphila I. This phenomenon might be because A.muciniphila I intervention significantly inhibited brown adipose tissue whitening and inflammation induced by HFD, by repairing the intestinal barrier and relieving endotoxemia. A.muciniphila II did not display the same results as A.muciniphila I in HFD mice, but had stronger effects in the NCD mice. Conclusions This study mainly reveals the distinct functions of different A.muciniphila genotypes on diet-induced obesity, suggesting that different A.muciniphila genotypes may play inequitable roles in pathological conditions through distinct action pathways.

\section{Background}

Obesity is a serious and growing global public health problem, with approximately 2 billion people around the world being overweight or obese[1]. Globally, more than 18\% of adults are expected to be obese by 2025[2]. Obesity is a serious clinical problem that increases the risk for many diseases, including insulin resistance, diabetes, hypertension, dyslipidemia, non-alcoholic fatty liver disease, and cardiovascular disease, and may result in high morbidity and mortality rates in the various metabolic diseases[3].

Mammals possess two specialized types of adipose tissue, white and brown adipose tissues, which serve opposite functions[4]. White adipose tissue (WAT) is characterized by large unilocular lipid-droplet-containing white adipocytes, and functions primarily to store dietary energy in the form of triglycerides in a single, large lipid droplet. White adipocytes are also known as unilocular adipocytes, while brown adipocytes are called multilocular adipocytes as they contain multiple small lipid droplets. Brown adipocytes are the main parenchymal cell type found in brown adipose tissue (BAT) and are characterized by large numbers of mitochondria, where uncoupling protein 1 (UCP1) possesses the ability to utilize free fatty acids and glucose for heat generation (non-shivering thermogenesis) [4]. In recent years, studies have found that BAT activity is negatively correlated with body mass index (BMI) [5]. In normally fed mice maintained at ambient temperature, white and brown adipocytes are found together in several subcutaneous and visceral depots[6-9]. In contrast, in the adipose organs of obese mice and humans, typical brown adipocytes were rarely detected, with most of them gradually replaced by unilocular adipocytes, a process known as BAT whitening[6, 10,11]. Studies have shown that BAT knockout in mice leads to obesity[12], while transplantation of BAT improves glucose tolerance, reduced weight and inhibits atherosclerosis in apoE ${ }^{-/-}$mice[13]. Increasingly, research has suggested that brown adipocytes have anti-obesity potential. 
Akkermansia muciniphila (A.muciniphila) is the only mammalian anaerobic bacteria in Verrucomicrobia that can be cultured in vitro, and was isolated successfully in 2004[14]. Many studies have found that the abundance of A.muciniphila was decreased in obese and type 2 diabetes models[15], while animal experiments found that A.muciniphila gavage could reduce obesity induced by a high-fat diet in mice and improve metabolic disorders, including hyperglycemia, insulin resistance and hyperlipidemia. However, the related mechanisms are still being explored[15-17]. Previous studies have shown a positive correlation between BAT marker genes and the abundance of A.muciniphila in the guts of HFD mice[16], suggesting an interaction between the two. In the present study, we explored the direct action of A.muciniphila intervention on enhancing or maintaining the activity of BAT in HFD mice.

There have been many studies regarding A.muciniphila, but their conclusions are somewhat contradictory[18]. Some researchers have suggested that A.muciniphila is a candidate probiotic, while others have found A.muciniphila levels were increased in the intestines of patients with Parkinson's disease, colon cancer and other diseases, indicating that A.muciniphila might be a harmful bacterium[19]. Some studies observed that different strains of the same bacteria do not necessarily play the same or similar roles, but there were some opposed roles[20]. We previously characterized the genomic architecture of A.muciniphila based on whole-genome sequencing, assembling, and annotation of 39 isolates derived from human and mouse feces, and divided them into three genotypes, including A.muciniphila I, II, and III [21, 22]. Phylogenetic analysis identified three specieslevel A.muciniphila phylogroups exhibiting distinct metabolic and functional features, and we found that the abundances of the different A.muciniphila genotypes varied in diabetic patients[21]. Therefore, in this context, the present study aimed mainly to explore the differences of the different A.muciniphila genotypes on regulating body metabolism, and verify whether A.muciniphila could improve glucose and lipid metabolism by activating or maintaining the activity of BAT, to reveal a new pathway of A.muciniphila in regulating metabolism.

\section{Methods}

Animals 60 Male age-matched C57BL/ 6 mice were purchased from the Southern Medical University Laboratory Animal Center. Mice were maintained on a 12:12h light-dark cycle with free access to water and standard chow (10\% Kcal from fat; Guangdong Medical Laboratory Animal Center, Guangzhou, China) or high-fat diet (60\% Kcal from fat; Guangdong Medical Laboratory Animal Center) starting from 8 weeks of age. The high-fat diet group and normal control diet group also received A.muciniphila I, A.muciniphila II or PBS intervention. C57BL/ 6 mice were orally gavaged with A muciniphila $(5 \times 109 \mathrm{cfu})$ in $200 \mu \mathrm{L}$ sterile PBS or $200 \mu \mathrm{L}$ sterile PBS daily during the dietary supplement. Body weights and food intake were determined weekly. The animal experiment lasted for 16 weeks. After 16 weeks of A.muciniphila administration, mice were fasted overnight and euthanized with cervical dislocation after intraperitoneal injection of pentobarbital sodium. Tissues (scapular, inguinal and epididymal fat, liver, and colon) were isolated and preserved after blood collection. All in vivo studies were authorized by and performed according to the Southern Medical University Animal Care and Use Committee. Identification of Akkermansia muciniphila PCR was performed using A.muciniphila in glycerol as the template and Premix Taq (Takara, Japan) on a BIO-RAD (Hercules, CA, USA) thermocycler. The A.muciniphila I and II primers are shown in Table 1. PCR was performed at $94^{\circ} \mathrm{C}$ for $2 \mathrm{~min}, 40$ cycles of denaturing at $94^{\circ} \mathrm{C}$ for $30 \mathrm{~s}$, annealing at $60^{\circ} \mathrm{C}$ for 30 s and extension at $72^{\circ} \mathrm{C}$ for $1 \mathrm{~min}$. A final extension at $72^{\circ} \mathrm{C}$ was performed 
for $5 \mathrm{~min}$ and the products held at $4^{\circ} \mathrm{C}$. The PCR products were mixed with 10xLoading buffer, and the samples were loaded in $10 \mu \mathrm{L}$ for agarose gel electrophoresis. The electrophoresis (120V, $60 \mathrm{~min}$ ) was stopped when the bromophenol blue ran to the bottom of the gel. The gel was photographed using an automatic gel imaging system. The molecular weight of A.muciniphila I and II amplification products was determined according to an included DNA marker. Culture and administration of Akkermansia muciniphila Akkermansia muciniphila I (GP01) and II (GP25) were cultured anaerobically in BHI (brainheart-infusion) broth (OXOID, England) supplemented with $0.5 \%$ porcine mucin (Sigma, Germany). After 48 hours of culture, the medium was removed and centrifuged at $3000 \mathrm{rpm}$ for 10 minutes. The remaining supernatant was removed and the precipitate was resuspended with sterile PBS and centrifuged again at $3000 \mathrm{rpm}$ for 10 minutes. The supernatant was again removed, the pellet was resuspended in sterile PBS and the suspension was used for mouse gavage. The concentration of bacteria was calculated by measuring the absorbance at the wavelength of $600 \mathrm{~nm}$. Oral glucose tolerance test A week before the mice were sacrificed, we conducted the OGTT experiment. C57BL/ 6 mice were fasted overnight for 12 hours, fasting blood glucose was measured via tail-cutting methods, and glucose $(2 \mathrm{~g} / \mathrm{kg})$ gavage was performed. Blood glucose was determined with a glucometer (Abbott Laboratories, Chicago, IL, USA) at 15, 30, 60, 90, $120 \mathrm{~min}$ after the glucose gavage. Biochemical Assays and ELISA After 16 weeks of A.muciniphila administration, the mice were anesthetized for blood collection. The serum was isolated and preserved for biochemical testing, and the mice were sacrificed. Serum levels of total cholesterol (TC) and triglyceride (TG) were detected using a Roche Modular P800 Analyzer (Roche, Switzerland). Serum levels of lipopolysaccharide (CUSABIO, China) and insulin (ALPCO, Salem, NH, USA) were analyzed with ELISA kits, following the manufacturers' instructions. Histology Adipose and colon tissues were fixed in $4 \%$ paraformaldehyde for $24 \mathrm{~h}$ at room temperature and immersed in $100 \%$ ethanol for $24 \mathrm{~h}$ before processing for paraffin embedding. Paraffin-embedded tissue sections (5- $\mu \mathrm{m}$ thickness) were stained with hematoxylin and eosin (H\&E) or Periodic acid-Schiff (PAS). For lipid staining, Oil Red $\mathrm{O}$ staining was performed according to the BioVision manual. In brief, liver samples were fixed at room temperature with $4 \%$ paraformaldehyde for 24 hours, dehydrated, embedded with OCT, and sliced using the freeze slicer. The slices were stained at $37^{\circ} \mathrm{C}$ with Oil Red $\mathrm{O}$ (Servicebio, China) solution for $15 \mathrm{~min}$. Nuclei were counterstained with hematoxylin for $10 \mathrm{~min}$, mounted with glycerol, and examined under a light microscope. The diameters of inguinal adipocytes and epididymal adipocytes were quantified by Image $J$ software. ImagePro Plus 6.0 software was used to analyze the number of colonic mucus cells and the degree of liver fat accumulation. Quantitative real-Time PCR and Western Blot Analysis For analysis of thermogenic and inflammatory genes in adipose tissue, total RNA was extracted by Trizol (Invitrogen, Carlsbad, CA, USA) and reverse transcribed into cDNA using the PrimeScript $^{\text {TM }}$ RT reagent Kit with gDNA Eraser (Takara, Japan). Quantitative real-time PCR was performed on a LightCycler 480 (Roche, Switzerland) using SYBR Green master mix (Takara, Japan). PCR parameters were $95^{\circ} \mathrm{C}$ for $30 \mathrm{~s}$, then 40 cycles of denaturing at $95^{\circ} \mathrm{C}$ for $5 \mathrm{~s}$ and annealing at $60{ }^{\circ} \mathrm{C}$ for $30 \mathrm{~s}, 1$ cycle of melting at $95^{\circ} \mathrm{C}$ for $5 \mathrm{~s}$ and $60^{\circ} \mathrm{C}$ for $1 \mathrm{~min}$. A final cooling at $50{ }^{\circ} \mathrm{C}$ for $30 \mathrm{~s}$ was performed. The results were normalized against the GAPDH gene. For analysis of A.muciniphila abundance in feces, fecal bacterial DNA was extracted using Magen Hipure Stool DNA kit (Magen, China). After adjusting the DNA concentration, real-time PCR was performed, using the same parameters 
used for thermogenic and inflammatory genes. The results were normalized to the $16 \mathrm{~S}$ rRNA gene. Primer sequences are shown in Table 1. Relative quantification was calculated using the 2- $\Delta \Delta \mathrm{Ct}$ method. For western blot analysis, protein was extracted from mouse tissues using RIPA buffer (Abcam, England) containing $0.5 \%$ NP40, $0.1 \%$ sodium deoxycholate, $150 \mathrm{mM} \mathrm{NaCl}, 50 \mathrm{mM}$ TrisHCl(7.4) and PMSF protease inhibitor (Abcam, England). Equal amounts of proteins were separated by SDS-PAGE and transferred to a PVDF membrane. The membrane was blocked in $5 \%$ non-fat milk for $1 \mathrm{~h}$ at room temperature and incubated in primary antibodies (UCP1, 1:1000, Abcam, England; occluding, 1:1000, Thermo Scientific, USA; $\beta$-tubulin, 1:500, Abcam, England; $\beta$-actin, 1:1000, Affinity, China) overnight at $4^{\circ} \mathrm{C}$. The membrane was washed 5 times ( 5 minutes per wash) in TBST and incubated with secondary antibody (anti-Rabbit-HRP conjugate 1:3000, anti-Mouse-HRP conjugate 1:3000, Abcam, England, the secondary antibody was selected according to the species origin of the primary antibody) for $1 \mathrm{~h}$. Following $5 \times 5 \mathrm{~min}$ washing in TBST, the protein bands were visualized with SuperSignalTM West Pico PLUS Chemiluminescent Substrate (Thermo Scientific, USA) and quantified using ImagePro Plus 6.0 software. Immunohistochemistry For UCP1 staining, tissue sections were repaired with antigen repair solution in a microwave oven and treated with $3 \%$ hydrogen peroxide solution for $25 \mathrm{~min}$ in the dark to quench endogenous peroxidase activity. The sections were covered uniformly with $3 \% \mathrm{BSA}$ and incubated at room temperature for $30 \mathrm{~min}$. The sections were incubated with rabbit anti-mouse UCP1 antibody (1:500, Abcam, England) overnight at $4^{\circ} \mathrm{C}$. The sections were washed thrice ( 5 min per wash) with PBS and incubated with goat anti-rabbit secondary antibodies (1:200, Servicebio, China) at room temperature for 50min. The sections were washed again three times with PBS and allowed to dry slightly. DAB color solution was added and the color developing time was controlled by microscope observation. Finally, hematoxylin was used to re-dye the nucleus and the section was dehydrated and sealed with neutral gum. The UCP1 content in the scapular brown adipose tissue was determined using ImagePro Plus 6.0 software. Statistical analysis. Data were expressed as means \pm s.e.m. Differences between two groups were assessed using the unpaired two-tailed Student's t-test and differences between three or more groups were assessed using one-way ANOVA. Data were analyzed using GraphPad Prism version 5.0 for Windows. Statistical comparisons were indicated with *, **, *** for $P<0.05, P<0.01$ and $P<0.001$ respectively.

\section{Results}

\section{The identification and abundance of Akkermansia muciniphila}

Before the beginning of the animal experiment, we identified A.muciniphila I and II in bacterial DNA from mouse feces, and these strains were independently isolated and preserved. After PCR amplification of A.muciniphila with specific primers designed for A.muciniphila I and II, we observed that the molecular weights of these two types of DNA amplicons were completely different, and that A.muciniphila I could be clearly distinguished from A.muciniphila II (Fig. 1A). In addition, before the intervention experiment, mouse fecal samples were collected, and the abundance of A.muciniphila I and II were analyzed by realtime PCR. The results showed that there was no significant difference in the original abundance of A.muciniphila I and II in the gut of the mice in each group (Fig. 1B and 1C). 
Akkermansia muciniphila effectively improved impaired fasting blood glucose and glucose homeostasis in HFD mice

We investigated the effects of A.muciniphila on glucose metabolism in HFD mice. Consistent with previous studies, fasting blood glucose was higher in HFD mice, and OGTT tests showed an increase in AUC (Fig. 2A-2C). Compared with the PBS treated group, A.muciniphila gavage decreased fasting blood glucose levels in HFD mice. OGTT tests indicated that A.muciniphila treatment obviously improved impaired glucose tolerance in HFD mice. We measured the serum insulin levels between HFD groups, which was found to be higher after A.muciniphila treatment (Fig. 2D), indicating that A.muciniphila might be able to promote the release of insulin to lower blood glucose. But only A.muciniphila I gavage showed significance in these results, while A.muciniphila II gavage did not play an equally effective role. No significant differences were found between the three NCD groups. Weight changes and food intake of the mice were recorded throughout the experiment (Fig. 2E and 2F). Gavage with A.muciniphila I and A.muciniphila II had no significant effect on the weight gain or food intake in either the HFD or NCD mice. These results suggested that A.muciniphila could improve glucose homeostasis in HFD mice, and that A.muciniphila I had a stronger effect than A.muciniphila II when compared against the PBS group.

\section{Akkermansia muciniphila relieved hyperlipidemia and fat accumulation in the livers of HFD mice}

We explored further the effects of A.muciniphila on lipid metabolism in HFD mice. HFD mice developed significant hyperlipidemia, evidenced by increasing levels of serum triglycerides (TG) and total cholesterol (TC) (Fig. 3A and 3B). A.muciniphila gavage improved the disordered lipid metabolism, observed as reduced serum TG and TC levels. In detail, A.muciniphila I showed obvious effects on both TG and TC, while A.muciniphila II improved only TC. Interestingly, A.muciniphila II showed a more significant difference when TC levels between the HFD groups were compared. As hyperlipidemia presumably results in fatty liver, we examined the pathological changes in the mouse livers. Liver weight was increased in HFD mice (Fig. 3C), and oil red O staining revealed severe accumulations of lipid drops in HFD mouse livers, which were evidently reduced after A.muciniphila gavage. However, only A.muciniphila I showed statistical significance (Fig 3D and 3E). Hyperlipidemia and fat accumulation in the liver were not obvious in NCD mice, and no significant differences were found among the three NCD groups. In summary, A.muciniphila I gavage was more effective than A.muciniphila II on alleviating hyperlipidemia and fat accumulation in in the livers of HFD mice.

\section{Akkermansia muciniphila diminished adipocyte size in WAT and relieved BAT whitening}

Considering the effects of A.muciniphila on glucose homeostasis and lipid metabolism, we explored the possibly related mechanisms. Brown adipose tissue activation was always accompanied by reduced blood glucose and lipid levels. We isolated the inguinal white adipose tissue (iWAT), epididymal white adipose tissue (eWAT) and scapular brown adipose tissue (BAT) of mice, performed H\&E staining. A high-fat diet significantly expanded the dimension of adipocytes in iWAT and eWAT (Fig. 4A and 4B), which was reduced after both A.muciniphila I and II gavage. A.muciniphila I had a more pronounced reduction in the diameter of inguinal adipocytes (Fig. 4D and 4E), but browning was not observed in iWAT or eWAT. We also found that scapular brown adipocytes in HFD mice changed from multilocular adipocytes to unilocular adipocytes, known as BAT whitening (Fig. 4C). Surprisingly, A.muciniphila gavage obviously relieved the whitening of BAT in HFD mice, showing significantly reduced numbers of unilocular adipocytes. There was, however, no way to compare the independent efficacy of A.muciniphila I and A.muciniphila II in HFD 
mice. Slight BAT whitening could be also seen in the three NCD groups, possibly related to the aging of the mice.

\section{Akkermansia muciniphila maintained BAT thermogenic activity and upregulated BAT markers in HFD mice}

In addition to morphological modifications, we investigated functional changes in BAT. Consistent with the above results, UCP1 staining showed that the protein content of UCP1 per area of BAT was increased in both A.muciniphila I and A.muciniphila II treated HFD mice, and that A.muciniphila II exhibited significant effects in NCD mice (Fig. 5E and 5F). As expected, the expression of UCP1 in BAT was increased after treatment with A.muciniphila, indicating increased thermogenic activity (Fig. 5A-5D). As shown in the western blot experiments, A.muciniphila I was more potent in HFD mice, while A.muciniphila II was more effective in NCD mice. At the transcription level, A.muciniphila I only played a role in HFD mice, and A.muciniphila II did not exhibit any statistical significance (Fig. 5G). We assessed the effects of A.muciniphila on BAT markers in addition to UCP1. Among the different markers of lipid metabolism involved in oxidation and thermogenic activity in BAT, we found that Cidea, Ppargc1a, Prdm16 and Pparg mRNA levels were observably increased after A.muciniphila gavage in HFD mice (Fig. 5H-5L). Meanwhile A.muciniphila II gavage did not exert a marked difference in Ppargc1a levels in HFD mice, but showed significant effects on Pparg expression levels in NCD mice. These results suggested that $A$.muciniphila could maintain thermogenic capacity in BAT and upregulated BAT markers. Furthermore, A.muciniphila I performed better in HFD mice, while A.muciniphila II showed marked improvements in NCD mice.

\section{Akkermansia muciniphila alleviated inflammation of brown adipose tissue in HFD mice}

Previous studies stated that local inflammation might down-regulate thermogenesis in BAT[23]. Conversely, reduction of BAT inflammation could maintain thermogenic activity. We analyzed the effects of $A$.muciniphila gavage on brown adipose tissue inflammation and measured several markers of metabolic inflammation in BAT, including II1b, Tnf, Emr1, Il6, $C c 12$, Lbp and Itgax. A.muciniphila I gavage markedly decreased the mRNA expression of macrophage inflammation markers (Itgax and Emr1), recruitment of immune cells (Ccl2) and LPS-binding protein $(L b p)$ in HFD mice, while A.muciniphila II gavage only significantly reduced Emr1 and $L b p$ transcript expression (Fig. 6D-6G). Il1b, Tnf and Il6 mRNA expression levels were not affected dramatically by A.muciniphila treatment in HFD mice (Fig. 6A-6C). In addition, A.muciniphila I gavage significantly reduced $L b p$ mRNA expression in NCD mice. These results indicated that Akk gavage could improve inflammation in BAT, and were regulated better under A.muciniphila I intervention.

\section{Akkermansia muciniphila improved endotoxemia and reversed the damage of intestinal barrier in HFD mice}

Local inflammation is inseparable from systemic inflammation, so we investigated whether A.muciniphila could relieve obesity-induced systemic chronic inflammation. The results showed lowered serum LPS levels in HFD mice, although A.muciniphila II did not show a significant decline (Fig. 7F). No significant differences were found between the three NCD groups. As the intestinal barrier determines susceptibility to systemic chronic inflammation, we further analyzed the number of colonic mucus cells and expression levels of tight junction protein after A.muciniphila intervention. PAS staining showed that the colonic mucus cells were decreased in HFD mice, but were restored to some extent after treatment with with both genotypes of A.muciniphila, (Fig. 7A and 7B). The expression of occludin in the colons of A.muciniphila treated HFD mice was higher compared to the PBS 
intervention group, A.muciniphila I and A.muciniphila II played similar roles (Fig. 7C and 7D). In contrast to the HFD mice, A.muciniphila gavage had no evident impact on colonic mucus cells or occludin expression in NCD mice (Fig. 7E). These results suggested that A.muciniphila gavage could relieve endotoxemia and the damaged intestinal barrier in HFD mice. A.muciniphila I and A.muciniphila II were both able to significantly repair the disturbed intestinal barrier, while only A.muciniphila I showed improvements against endotoxemia.

\section{Discussion}

A.muciniphila was first isolated and cultured in vitro by researchers in 2004 . As an intestinal symbiotic bacteria, it accounts for a considerable proportion of the intestinal microflora of mammals[14]. Studies have found that the abundance of A.muciniphila in the intestines of obese and type 2 diabetes patients was significantly reduced[15], while the administration of A.muciniphila to mice on a high-fat diet alleviated obesity, reduced weight gain, and improved blood glucose levels and insulin resistance[24]. Studies have also shown that the diabetes drug metformin improved glucose metabolism in HFD mice, to some extent, by increasing the abundance of A.muciniphila in the gut[25]. A series of studies suggested that A.muciniphila was a highly beneficial intestinal symbiont. However, researchers found that, in some type 2 diabetes, colon cancer and Parkinson's patients, the abundance of $A$.muciniphila increased, suggesting that $A$.muciniphila might play a part in these diseases and might be a harmful bacterium[19, 26-30]. There was no detailed explanation for what led to completely different, or even opposing, conclusions, as experts stated that whether a microbe is helpful or harmful is highly dependent on a multitude of host and microbial factors, including the dose, strain, duration of colonization, and specific application of the microbe; and the age, genetic background, environmental factors, disease status, and host process in question[13], and here our hypothesis was that this discrepancy might be related to different A.muciniphila genotypes.

We had previously isolated 39 strains of A.muciniphila from the human intestine, and, using high-throughput sequencing, divided them into three genotypes, of which the type strain ATCC-835 belongs to A.muciniphila I [21]. Consistent with previous studies, our study found that A.muciniphila abundance was significantly reduced in obese people (BMI 30), with a significant decrease in A.muciniphila I and A.muciniphila II. In 2014, Shin et al. [40] demonstrated that metformin could improve glucose homeostasis in HFD mice by increasing the abundance of A.muciniphila in the gut. However, we found that, in type 2 diabetic patients treated with metformin, the abundance of A.muciniphila I increased obviously while A.muciniphila II did not change significantly, which led us to speculate that the roles of A.muciniphila I and II might be different[21]. In the present study, we found that, in HFD mice, A.muciniphila I and II could improve the impaired glucose tolerance and promote the release of insulin to an extent. The effects of A.muciniphila II on regulating glucose metabolism were not obvious, however, suggesting that A.muciniphila gavage could ameliorate glucose metabolic disorders in mice, but that the effects of $A$.muciniphila I were more pronounced than A.muciniphila II .

Recent studies have shown that the lipid-lowering drugs ezetimibe, fenofibrate, and rusvarastine inhibit the progression of non-alcoholic steatohepatitis(NASH), indicating the role of hyperlipidemia in NASH[31]. Currently, there is a shortage of drugs to treat NASH correspondingly, therefore necessitating precautions against NASH. Our experiments found that A.muciniphila gavage significantly decreased the serum TG and TC levels in HFD mice, with A.muciniphila I being more effective in TG regulation, while A.muciniphila II was more effective in modulating TC levels. Additionally, we found that A.muciniphila gavage significantly inhibited the accumulation of fat in the liver while improving hyperlipidemia in 
HFD mice. However, only A.muciniphila I showed statistically significant effects. Compared with TC, TG should be a more appropriate independent risk factor for fatty liver. Studies have shown that serum TG was a significant risk factor for fatty liver[32], while TC was predominantly correlated with coronary atherosclerotic cardiopathy[33]. Given that A.muciniphila II had a more remarkable effect on TC, A.muciniphila II may act more strongly in regulating cholesterol metabolism. Meanwhile, A.muciniphila I reduced serum TG levels notably, which may have promoted the improvement of fatty liver.

A detailed analysis in 2015 showed that the abundance of A.muciniphila was positively correlated with BAT marker gene levels in HFD mice[16]. In 2017, Gao [18] proved that A.muciniphila administration could directly promote the browning of WAT. However, this study was performed by treating A.muciniphila culture medium, and therefore could not exclude the interference of A.muciniphila metabolites in the culture medium, and this study did not focus on changes in BAT itself[34]. Studies have shown that BAT happened to be whitened in cases of obesity, presenting as multilocular adipocytes gradually replaced by unilocular adipocytes, increased lipid droplets in BAT, decreased numbers of mitochondria and UCP1 expression, infiltrating inflammatory factors, and decreased levels of BAT marker genes [23]. Other studies found that BAT knockout in mice could lead to obesity[12], that reduced BAT-dependent non-shivering thermogenesis could damage insulin sensitivity, and the expression of UCP1 in WAT effectively reduced weight[35, 36]. Several findings suggested that BAT whitening and BAT dysfunction might induce or even exacerbate obesity and related metabolic syndromes[3]. In this study, it was demonstrated that the scapular BAT was significantly whitened in HFD mice, and the thermogenic activity was decreased. Strikingly, after A.muciniphila treatment, although we did not observe significant browning of iWAT and eWAT, the number of unilocular adipocytes decreased significantly in BAT, UCP1 expression increased and other BAT marker gene levels were upregulated. We found that A.muciniphila can inhibit BAT whitening and maintain BAT activity in HFD mice, although the effects of A.muciniphila II were slightly inferior to A.muciniphila I. In addition to obesity, aging can also lead to BAT whitening $[37,38]$. It is worth mentioning that we observed mild BAT whitening in the NCD mice, which might be due to aging and A.muciniphila I had no distinct effect. Nevertheless, A.muciniphila II could play a considerable role. These observations led us to suspect that the mechanisms of these two genotypes may be widely divergent.

In obesity, the intestinal barrier function is damaged, intestinal permeability increased, and the endotoxins secreted by gut microbiota are released into the blood, causing endotoxemia, which in turn leads to chronic systemic inflammation and further local inflammation[39, 40]. The results of this study showed that both A.muciniphila I and II effectively increased colon mucus cells and tight junction protein expression. Correspondingly, after A.muciniphila I treatment, HFD mice showed reduced serum LPS and reduced transcriptional levels of inflammatory genes in BAT, while A.muciniphila II showed no difference, suggesting that A.muciniphila I can relieve both systemic and local inflammation. Given that BAT local inflammation negatively regulates thermogenesis[23], this may explain why A.muciniphila I can maintain BAT activity and inhibit BAT whitening. To conclude, A.muciniphila I may improve endotoxemia induced by HFD by repairing the intestinal barrier, thereby inhibiting the development of inflammation in BAT and maintaining the activity of BAT. BAT can make use of the free fatty acid and glucose in blood as the substrate for thermogenesis[41], so the maintainance of BAT activity is advantageous to removing excessive free fatty acid and glucose. This, in turn, reduces blood glucose and lipid levels, improving the body's glucose and lipid metabolism (Fig. 8). This explains why A.muciniphila I gavage alleviated the impaired glucose tolerance, hyperlipidemia and liver fat accumulation in HFD mice. 
It is worth noting that although A.muciniphila II could also repair the intestinal barrier, the effect of A.muciniphila II on endotoxemia was not apparent, leading to a failure of reversing BAT inflammation. Endotoxemia is not only associated with intestinal barrier function, but also with gut microbiome structure. For example, Cani proved that plasma LPS concentrations correlated negatively with Bifidobacterium spp[42, 43]. Studies have also demonstrated that high plasma LPS levels could result from an increased production of endotoxins by a change in gut microbiota[43, 44]. Despite the evidence that both of A.muciniphila I and A.muciniphila II can repair the intestinal barrier, they do not both improve endotoxemia, possibly because they possess different functions in the intestinal microecology. Despite A.muciniphila II not effectively improving BAT inflammation in HFD mice, it did function manifestly in alleviating BAT whitening and maintaining BAT activity, and acted in NCD mice (Fig. 9). It follows that the action pathways of these two genotypes may not be consistent, but further studies are needed to prove this hypothesis. Our results suggest A.muciniphila I and II exert different impacts on blood glucose and lipid metabolism, which may be related to their specific target pathways. Disparate mechanisms may determine disparate roles, which may be the cause of inconsistent evaluation of different A.muciniphila genotypes.

\section{Conclusions}

In mice, A.muciniphila intervention can repair the intestinal barrier damage induced by a high-fat diet, alleviate endotoxemia and lower the degree of BAT inflammation, thus relieving BAT whitening, improving glucose tolerance and hyperlipidemia, and reducing liver fat accumulation in HFD mice. A.muciniphila I showed greater effectiveness in HFD mice, while A.muciniphila II produced greater effects in NCD mice. This study reveals the differences between the different A.muciniphila genotypes in improving metabolism, suggesting that different A.muciniphila genotypes may play different roles in the same disease state, which partly explains the controversy over the functions of A.muciniphila in previous studies. In addition, the present study found that A.muciniphila intervention exuded an inhibitory effect on BAT inflammation and whitening, providing a novel mechanistic direction for future research on the regulation of body metabolism by A.muciniphila, which is conducive to a deeper understanding of A.muciniphila action.

\section{Abbreviations}

Akkermansia muciniphila: A. muciniphila; HFD: high-fat diet; NCD: normal chow diet; WAT: white adipose tissue; BAT: brown adipose tissue; UCP1: uncoupling protein 1; BMI: body mass index; BHI: brain-heartinfusion; OGTT: oral glucose tolerance test; TC: total cholesterol; TG: triglyceride; H\&E: hematoxylin and eosin; PAS: Periodic acid-Schiff; iWAT: inguinal white adipose tissue; eWAT: epididymal white adipose tissue; Lbp: LPS-binding protein; NASH: non-alcoholic steatohepatitis.

\section{Declarations}

\section{Ethics approval and consent to participate}

All in vivo studies involving animals were authorized by and performed according to the Southern Medical University Animal Care and Use Committee.

Consent for publication 
Not applicable.

\section{Availability of data and materials}

All data generated or analysed during this study are included in this published article.

\section{Competing interests}

The authors declare that they have no competing interests.

\section{Funding}

This study was funded by grants from the Guangdong Provincial Department of Science and Technology (2015A020213002), and the Guangzhou Kangze Medical Science and Technology Co., Ltd Fund (201511). The funders played a supervisory and management role in this study.

\section{Authors' Contributions}

LD and ZO designed and performed the experiments, analyzed data, and wrote the paper. DQ H, CL, ZL, FFW, CHN and JG helped dissect mice. WTL helped to isolate brown adipose tissue. YZP designed the experiments, wrote the paper, and supervised the project.

\section{Acknowledgment}

Not applicable.

\section{References}

1. Asrani SK, Devarbhavi H, Eaton J, Kamath PS. Burden of liver diseases in the world. J HEPATOL. [Journal Article; Review]. 2018 2018-09-26.

2. Trends in adult body-mass index in 200 countries from 1975 to 2014: a pooled analysis of 1698 populationbased measurement studies with 19.2 million participants. LANCET. [Journal Article; Meta-Analysis; Research Support, Non-U.S. Gov't; Review]. 2016 2016-04-02;387(10026):1377-96.

3. Kotzbeck P, Giordano A, Mondini E, Murano I, Severi I, Venema W, et al. Brown adipose tissue whitening leads to brown adipocyte death and adipose tissue inflammation. J LIPID RES. 2018 2018-01-01;59(5):78494.

4. Cinti S. Transdifferentiation properties of adipocytes in the adipose organ. AM J PHYSIOL-ENDOC M. 2009;297(5) :E977-86.

5. Wang X, Minze LJ, Shi ZZ. Functional imaging of brown fat in mice with 18F-FDG micro-PET/CT. J Vis Exp. [Journal Article; Video-Audio Media]. 2012 2012-11-23(69).

6. Cinti S. The adipose organ. Prostaglandins Leukot Essent Fatty Acids. [Journal Article; Review]. 2005 200507-01;73(1):9-15.

7. Cannon B, Nedergaard J. Brown adipose tissue: function and physiological significance. PHYSIOL REV. [Journal Article; Research Support, Non-U.S. Gov't; Review]. 2004 2004-01-01;84(1):277-359. 
8. Murano I, Barbatelli G, Giordano A, Cinti S. Noradrenergic parenchymal nerve fiber branching after cold acclimatisation correlates with brown adipocyte density in mouse adipose organ. J ANAT. [Journal Article; Research Support, Non-U.S. Gov't]. 2009 2009-01-01;214(1):171-8.

9. Vitali A, Murano I, Zingaretti MC, Frontini A, Ricquier D, Cinti S. The adipose organ of obesity-prone C57BL/6J mice is composed of mixed white and brown adipocytes. J LIPID RES. [Comparative Study; Journal Article; Research Support, Non-U.S. Gov't]. 2012 2012-04-01;53(4):619-29.

10. Sbarbati A, Morroni M, Zancanaro C, Cinti S. Rat interscapular brown adipose tissue at different ages: a morphometric study. Int J Obes. [Journal Article; Research Support, Non-U.S. Gov't]. 1991 1991-0901;15(9):581-7.

11. Cinti S, Frederich RC, Zingaretti MC, De Matteis R, Flier JS, Lowell BB. Immunohistochemical localization of leptin and uncoupling protein in white and brown adipose tissue. ENDOCRINOLOGY. [Journal Article; Research Support, Non-U.S. Gov't; Research Support, U.S. Gov't, P.H.S.]. 1997 1997-02-01;138(2):797-804.

12. Lowell BB, S-Susulic V, Hamann A, Lawitts JA, Himms-Hagen J, Boyer BB, et al. Development of obesity in transgenic mice after genetic ablation of brown adipose tissue. NATURE. [Journal Article; Research Support, Non-U.S. Gov't; Research Support, U.S. Gov't, P.H.S.]. 1993 1993-12-23;366(6457):740-2.

13. Kikai M, Yamada H, Wakana N, Terada K, Yamamoto K, Wada N, et al. Transplantation of brown adipose tissue inhibits atherosclerosis in apoE-/- mice: contribution of the activated FGF-21-adiponectin axis. CARDIOVASC RES. [Journal Article]. 2018 2018-05-01;114(6):i1-13.

14. Derrien M, Belzer C, de Vos WM. Akkermansia muciniphila and its role in regulating host functions. Microb Pathog. [Journal Article; Review]. 2017 2017-05-01;106:171-81.

15. Everard A, Belzer C, Geurts L, Ouwerkerk JP, Druart C, Bindels LB, et al. Cross-talk between Akkermansia muciniphila and intestinal epithelium controls diet-induced obesity. Proc Natl Acad Sci U S A. [Journal Article; Research Support, Non-U.S. Gov't]. 2013 2013-05-28;110(22):9066-71.

16. Schneeberger M, Everard A, Gómez-Valadés AG, Matamoros S, Ramírez S, Delzenne NM, et al. Akkermansia muciniphila inversely correlates with the onset of inflammation, altered adipose tissue metabolism and metabolic disorders during obesity in mice. SCI REP-UK. 2015;5(1).

17. Dao MC, Everard A, Aron-Wisnewsky J, Sokolovska N, Prifti E, Verger EO, et al. Akkermansia muciniphila and improved metabolic health during a dietary intervention in obesity: relationship with gut microbiome richness and ecology. GUT. 2016;65(3):426-36.

18. Zhai Q, Feng S, Arjan N, Chen W. A next generation probiotic, Akkermansia muciniphila. Crit Rev Food Sci Nutr. [Journal Article]. 2018 2018-10-29:1-10.

19. Cirstea M, Radisavljevic N, Finlay BB. Good Bug, Bad Bug: Breaking through Microbial Stereotypes. CELL HOST MICROBE. 2018 2018-01-01;23(1):10-3.

20. Geva-Zatorsky N, Sefik E, Kua L, Pasman L, Tan TG, Ortiz-Lopez A, et al. Mining the Human Gut Microbiota for Immunomodulatory Organisms. CELL. [Journal Article; Research Support, U.S. Gov't, Non-P.H.S.; Research Support, Non-U.S. Gov't]. 2017 2017-02-23;168(5):928-43.

21. Guo X, Li S, Zhang J, Wu F, Li X, Wu D, et al. Genome sequencing of 39 Akkermansia muciniphila isolates reveals its population structure, genomic and functional diverisity, and global distribution in mammalian gut 
microbiotas. BMC GENOMICS. [Journal Article]. 2017 2017-10-18;18(1):800.

22. Guo X, Zhang J, Wu F, Zhang M, Yi M, Peng Y. Different subtype strains of Akkermansia muciniphila abundantly colonize in southern China. J APPL MICROBIOL. [Journal Article; Research Support, Non-U.S. Gov't]. 2016 2016-02-01;120(2):452-9.

23. Martins FF, Bargut TCL, Aguila MB, Mandarim-de-Lacerda CA. Thermogenesis, fatty acid synthesis with oxidation, and inflammation in the brown adipose tissue of ob/ob (-/-) mice. Annals of Anatomy Anatomischer Anzeiger. 2017;210:44-51.

24. Plovier H, Everard A, Druart C, Depommier C, Van Hul M, Geurts L, et al. A purified membrane protein from Akkermansia muciniphila or the pasteurized bacterium improves metabolism in obese and diabetic mice. NAT MED. 2017;23(1):107-13.

25. Shin N, Lee J, Lee H, Kim M, Whon TW, Lee M, et al. An increase in theAkkermansia spp. population induced by metformin treatment improves glucose homeostasis in diet-induced obese mice. GUT. 2014;63(5):727-35.

26. Heintz-Buschart A, Pandey U, Wicke T, Sixel-Doring F, Janzen A, Sittig-Wiegand E, et al. The nasal and gut microbiome in Parkinson's disease and idiopathic rapid eye movement sleep behavior disorder. Mov Disord. [Journal Article]. 2018 2018-01-01;33(1):88-98.

27. Berer K, Gerdes LA, Cekanaviciute E, Jia X, Xiao L, Xia Z, et al. Gut microbiota from multiple sclerosis patients enables spontaneous autoimmune encephalomyelitis in mice. Proc Natl Acad Sci U S A. [Journal Article; Research Support, N.I.H., Extramural; Research Support, Non-U.S. Gov't; Research Support, U.S. Gov't, Non-P.H.S.; Twin Study]. 2017 2017-10-03;114(40):10719-24.

28. Cekanaviciute E, Yoo BB, Runia TF, Debelius JW, Singh S, Nelson CA, et al. Gut bacteria from multiple sclerosis patients modulate human T cells and exacerbate symptoms in mouse models. Proc Natl Acad Sci U S A. [Journal Article; Research Support, N.I.H., Extramural; Research Support, Non-U.S. Gov't; Research Support, U.S. Gov't, Non-P.H.S.]. 2017 2017-10-03;114(40):10713-8.

29. Vogt NM, Kerby RL, Dill-McFarland KA, Harding SJ, Merluzzi AP, Johnson SC, et al. Gut microbiome alterations in Alzheimer's disease. Sci Rep. [Journal Article]. 2017 2017-10-19;7(1):13537.

30. Qin J, Li Y, Cai Z, Li S, Zhu J, Zhang F, et al. A metagenome-wide association study of gut microbiota in type 2 diabetes. NATURE. [Journal Article; Research Support, Non-U.S. Gov't]. 2012 2012-10-04;490(7418):55-60.

31. Orime K, Shirakawa J, Togashi Y, Tajima K, Inoue H, Nagashima Y, et al. Lipid-lowering agents inhibit hepatic steatosis in a non-alcoholic steatohepatitis-derived hepatocellular carcinoma mouse model. EUR J PHARMACOL. 2016;772:22-32.

32. Ma Y, Zhao L, Xian X, Yang D, Huang W, Wang Y, et al. A case-control study on the relationship between HDL2b and non-alcoholic fatty liver disease in Chinese type 2 diabetic patients. CLIN CHEM LAB MED. 2009;47(9).

33. Gallo A, Giral P, Carrie A, Carreau V, Beliard S, Bittar R, et al. Early coronary calcifications are related to cholesterol burden in heterozygous familial hypercholesterolemia. J CLIN LIPIDOL. [Journal Article]. 2017 2017-05-01;11(3) :704-11. 
34. Gao X, Xie Q, Kong P, Liu L, Sun S, Xiong B, et al. Polyphenol- and Caffeine-Rich Postfermented Pu-erh Tea Improves Diet-Induced Metabolic Syndrome by Remodeling Intestinal Homeostasis in Mice. INFECT IMMUN. 2017 2017-10-23;86(1).

35. Roberts-Toler C, O'Neill BT, Cypess AM. Diet-induced obesity causes insulin resistance in mouse brown adipose tissue. Obesity (Silver Spring). [Journal Article; Research Support, N.I.H., Extramural; Research Support, N.I.H., Intramural]. 2015 2015-09-01;23(9):1765-70.

36. Kopecky J, Clarke G, Enerback S, Spiegelman B, Kozak LP. Expression of the mitochondrial uncoupling protein gene from the aP2 gene promoter prevents genetic obesity. J CLIN INVEST. [Comparative Study; Journal Article; Research Support, Non-U.S. Gov't; Research Support, U.S. Gov't, P.H.S.]. 1995 1995-1201;96(6):2914-23.

37. Palmer AK, Kirkland JL. Aging and adipose tissue: potential interventions for diabetes and regenerative medicine. EXP GERONTOL. 2016;86:97-105.

38. Rogers NH, Landa A, Park S, Smith RG. Aging leads to a programmed loss of brown adipocytes in murine subcutaneous white adipose tissue. AGING CELL. 2012;11(6):1074-83.

39. Brun P, Castagliuolo I, Leo VD, Buda A, Pinzani M, Palù G, et al. Increased intestinal permeability in obese mice: new evidence in the pathogenesis of nonalcoholic steatohepatitis. AM J PHYSIOL-GASTR L. 2007;292(2):G518-25.

40. Scalera A. What does irritable bowel syndrome share with non-alcoholic fatty liver disease? WORLD J GASTROENTERO. 2013;19(33):5402.

41. Bargut TCL, Aguila MB, Mandarim-de-Lacerda CA. Brown adipose tissue: Updates in cellular and molecular biology. Tissue and Cell. 2016;48(5):452-60.

42. Cani PD, Neyrinck AM, Fava F, Knauf C, Burcelin RG, Tuohy KM, et al. Selective increases of bifidobacteria in gut microflora improve high-fat-diet-induced diabetes in mice through a mechanism associated with endotoxaemia. DIABETOLOGIA. 2007 2007-10-01;50(11):2374-83.

43. Moreira APB, Texeira TFS, Ferreira AB, Do Carmo Gouveia Peluzio M, de Cássia Gonçalves Alfenas R. Influence of a high-fat diet on gut microbiota, intestinal permeability and metabolic endotoxaemia. BRIT J NUTR. 2012;108(05):801-9.

44. Cani PD, Bibiloni R, Knauf C, Waget A, Neyrinck AM, Delzenne NM, et al. Changes in gut microbiota control metabolic endotoxemia-induced inflammation in high-fat diet-induced obesity and diabetes in mice. DIABETES. [Journal Article; Research Support, Non-U.S. Gov't]. 2008 2008-06-01;57(6):1470-81.

\section{Table}

Table 1. 


\begin{tabular}{|l|l|l|}
\hline Gene & Forward(5'-3') & Reverse(5'-3') \\
\hline A.muciniphila & AGCCTGAACGCATTATCCGC & AGGGCAGTTCTTCCAGCGTA \\
\hline A.muciniphila & CGTCTGGTTCGGCTCCAAGTG & TGACCACGTACCATTGCGGAATG \\
\hline 16S rRNA & GTGYCAGCMGCCGCGGTAA & GGACTACNVGGGTWTCTAAT \\
\hline Ucp1 & AGGCTTCCAGTACCATTAGGT & CTGAGTGAGGCAAAGCTGATTT \\
\hline Cidea & TGACATTCATGGGATTGCAGAC & GGCCAGTTGTGATGACTAAGAC \\
\hline Prdm16 & TGCTGACGGATACAGAGGTGT & CCACGCAGAACTTCTCGCTAC \\
\hline Ppargc1a & TATGGAGTGACATAGAGTGTGCT & GTCGCTACACCACTTCAATCC \\
\hline Ppara & AACATCGAGTGTCGAATATGTGG & CCGAATAGTTCGCCGAAAGAA \\
\hline Pparg & GGAAGACCACTCGCATTCCTT & GTAATCAGCAACCATTGGGTCA \\
\hline II6 & CTGCAAGAGACTTCCATCCAG & AGTGGTATAGACAGGTCTGTTGG \\
\hline Tnf & CAGGCGGTGCCTATGTCTC & CGATCACCCCGAAGTTCAGTAG \\
\hline II1b & TTCAGGCAGGCAGTATCACTC & GAAGGTCCACGGGAAAGACAC \\
\hline Emr1 & CTCAGTCTGCACCAATATCCTG & CCACAGAGTTAGAGCAGTTGGAA \\
\hline CCI2 & TAAAAACCTGGATCGGAACCAAA & GCATTAGCTTCAGATTTACGGGT \\
\hline Lbp & GCAGCCGCATTTGTGATTTG & TTCGACTCGATCTGGTTGTGG \\
\hline Itgax & CCAAGACATCGTGTTCCTGATT & ACAGCTTTAACAAAGTCCAGCA \\
\hline GAPDH & AGGTCGGTGTGAACGGATTTG & TGTAGACCATGTAGTTGAGGTCA \\
\hline
\end{tabular}

\section{Figures}



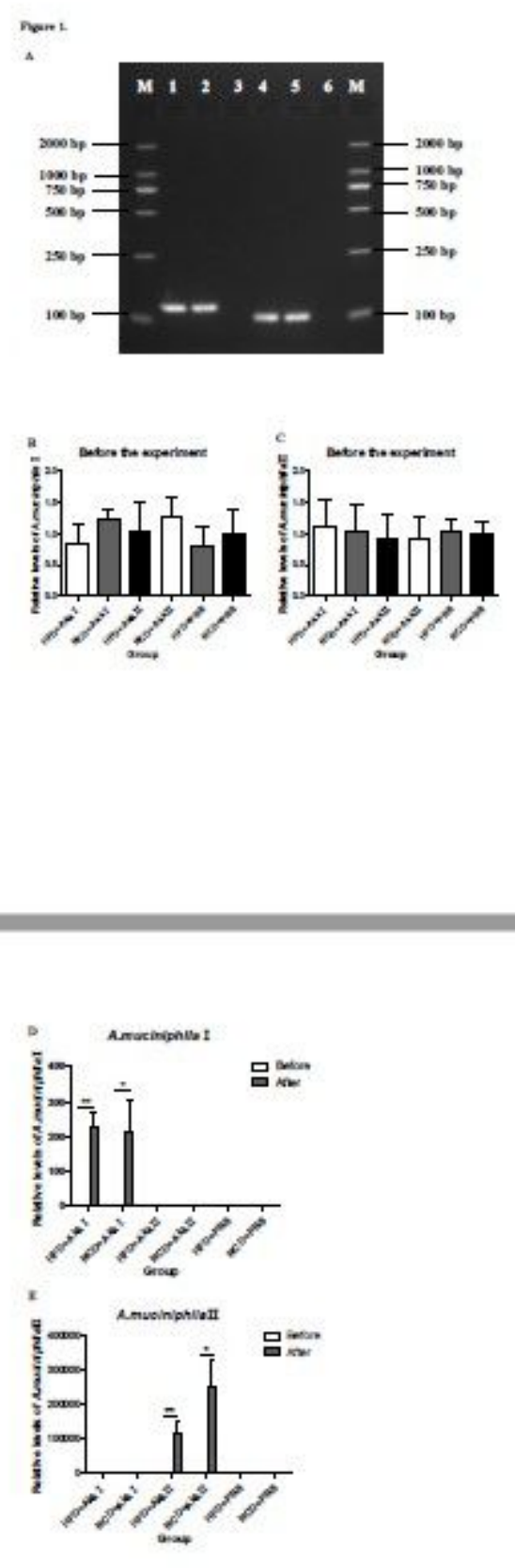

Figure 1

The identification and abundance of Akkermansia muciniphila (HFD+Akk I indicates high fat diet plus A.muciniphila I intervention; HFD+Akk II indicates high fat diet plus A.muciniphila II intervention; HFD+PBS indicates high fat diet plus PBS intervention; NCD+Akk I represents normal control diet plus A.muciniphila I intervention; NCD+Akk II represents normal control diet plus A.muciniphila II intervention; NCD+PBS indicates normal control diet plus PBS intervention). (A) DNA molecular weights of A.muciniphila I and II were determined by PCR amplification and agarose gel electrophoresis by designing specific primer sequences (Lane 1 and lane 2 were A.muciniphila I, and lane 3 was A.muciniphila I negative control; lane 4 and lane 5 were A.muciniphila II, and lane 6 was A.muciniphila II negative 
control). (B, C) The original abundance of A.muciniphila I and II in the mouse gut was measured before the beginning of animal experiment. Data are presented as mean \pm SEM with $n=5-8$ in each group. ${ }^{*}<<$ $0.05 ; * \star \mathrm{P}<0.01 ; * \star \star \mathrm{P}<0.001$.
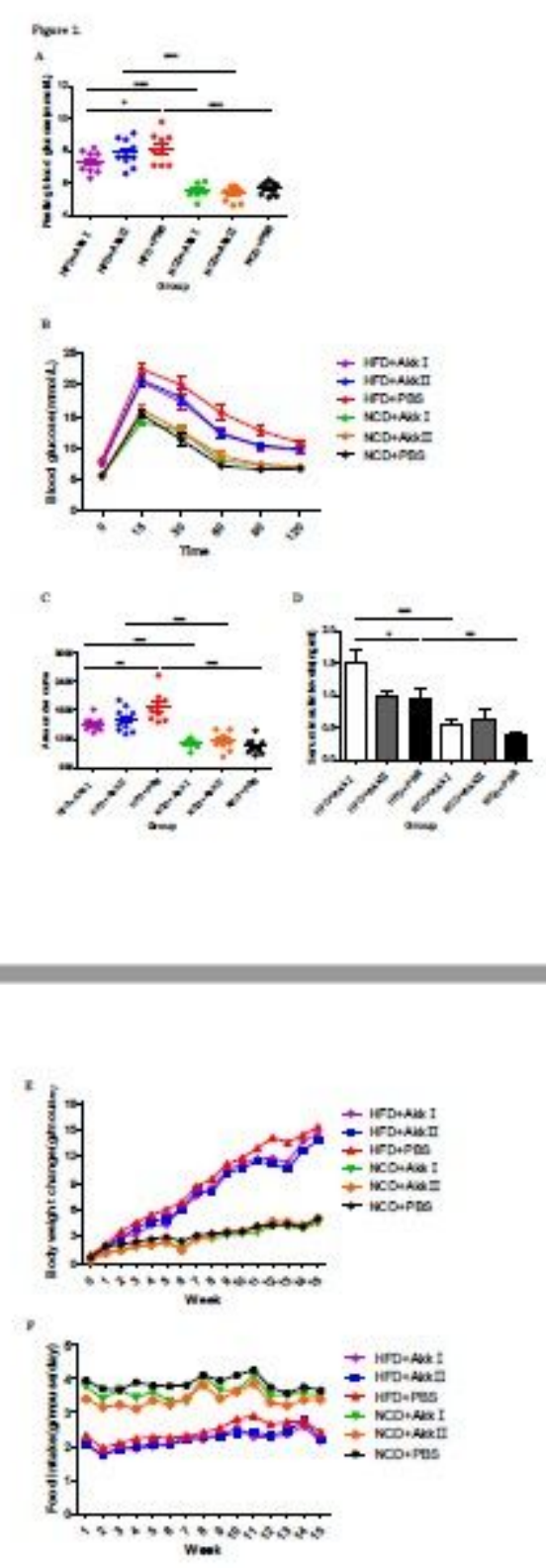

\section{Figure 2}

Akkermansia muciniphila effectively improved impaired fasting blood glucose and glucose homeostasis in HFD mice. (A) Fasting blood glucose and (B) OGTT tests were analyzed in all groups. According to the OGTT experiment, (C) the mean area under the curve (AUC) was determined and compared. (D) Insulin ELISA results are shown. During the whole process of animal experiments, body weight (E) and food 
intake per mice $(F)$ was recorded once a week. Data are presented as mean \pm SEM with $n=6-10$ in each group. ${ }^{\star} \mathrm{P}<0.05 ; * \star \mathrm{P}<0.01 ; * \star \star \mathrm{P}<0.001$.

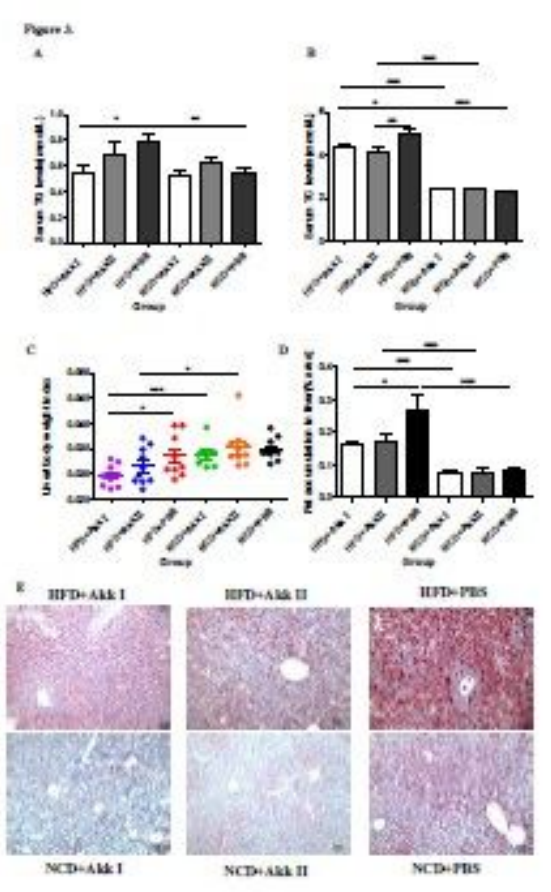

\section{Figure 3}

Akkermansia muciniphila relieved hyperlipidemia and fat accumulation in liver in HFD mice. (A) Serum TG and (B) TC levels in all mice were determined using a Roche automatic biochemical analyzer. The livers were weighed, and the lipid content in livers was analyzed by oil red $\mathrm{O}$ staining. (C) The ratio of liver to body weight per mouse and (E) representative pictures of Oil red $O$ stained liver sections are shown. (D) Liver lipid content was quantified using Image-Pro Plus 6.0 software. Data are presented as mean \pm SEM with $\mathrm{n}=6-10$ in each group. ${ }^{*} \mathrm{P}<0.05 ;{ }^{* * P}<0.01 ; * \star * \mathrm{P}<0.001$. 

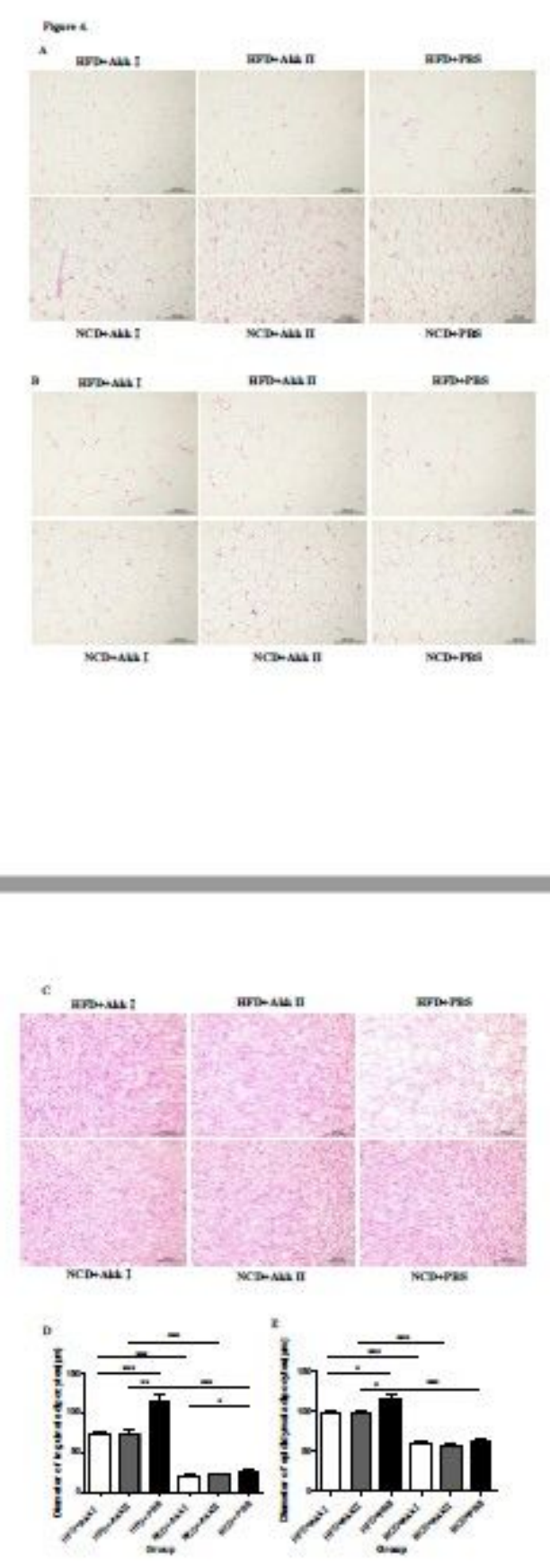

\section{Figure 4}

Akkermansia muciniphila diminished adipocyte size in WAT and relieved BAT whitening. After the adipose tissue was removed, H\&E staining was performed to analyze the size of white adipocytes and the morphological changes of brown adipocytes. Representative H\&E images of (A) iWAT, (B) eWAT and (C) BAT are shown. The diameters of inguinal adipocytes $(D)$ and epididymal adipocytes $(E)$ were quantified by Image $J$ software. Data are presented as mean \pm SEM with $n=5-10$ in each group. ${ }^{*} P<0.05 ; * \star P<0.01$; $\star \star \star P<0.001$. 

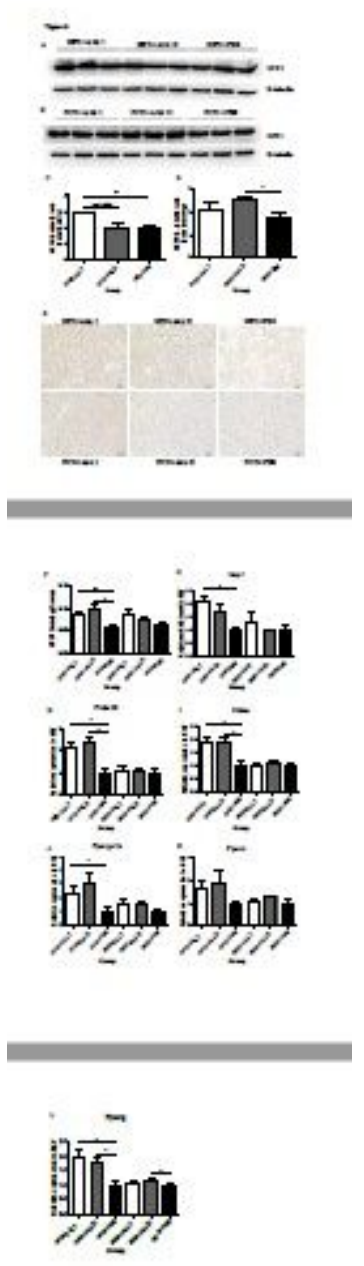

\section{Figure 5}

Akkermansia muciniphila maintained BAT thermogenic activity and upregulated BAT markers in HFD mice. Protein and RNA from brown adipose tissue were extracted for western blot and quantitative realtime PCR analyses, respectively. (A) Representative western blot of BAT lysates in HFD groups and (B) NCD groups are shown. (C, D) UCP1 expression was quantified using Image-Pro Plus 6.0 software and (G$\mathrm{L})$ the transcription levels of BAT markers were calculated. The UCP1 content of brown adipose tissue was analyzed by immunohistochemistry. (E) Representative images of UCP1 staining in BAT are shown. (F) The protein content of UCP1 per area was quantified using Image-Pro Plus 6.0 software. Data are presented as the mean \pm SEM with $n=3$ in each group. ${ }^{*} P<0.05 ; * \star P<0.01 ; * \star \star P<0.001$. 

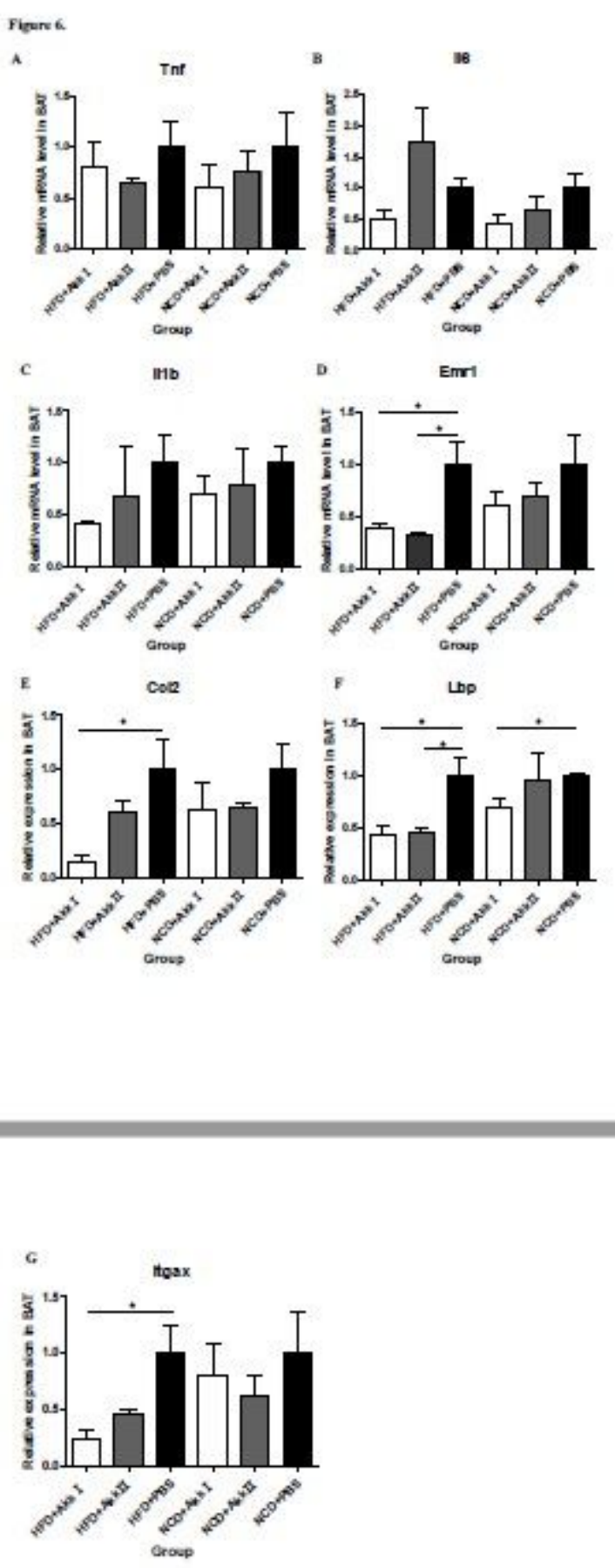

\section{Figure 6}

Akkermansia muciniphila alleviated inflammation of brown adipose tissue in HFD mice. To determine the local inflammation of brown adipose tissue, we analyzed the level of inflammatory genes in brown adipose tissue by quantitative real-time PCR. (A-G) The relative mRNA abundance of Tnf, Il6 $\| 11 \mathrm{~b}$, Emr1,Ccl2, Lbp and Itgax in 6 groups was calculated. Data are presented as the mean \pm SEM with $n=3$ in each group. ${ }^{*} \mathrm{P}<0.05 ;{ }^{* \star} \mathrm{P}<0.01 ;{ }^{* \star \star} \mathrm{P}<0.001$. 

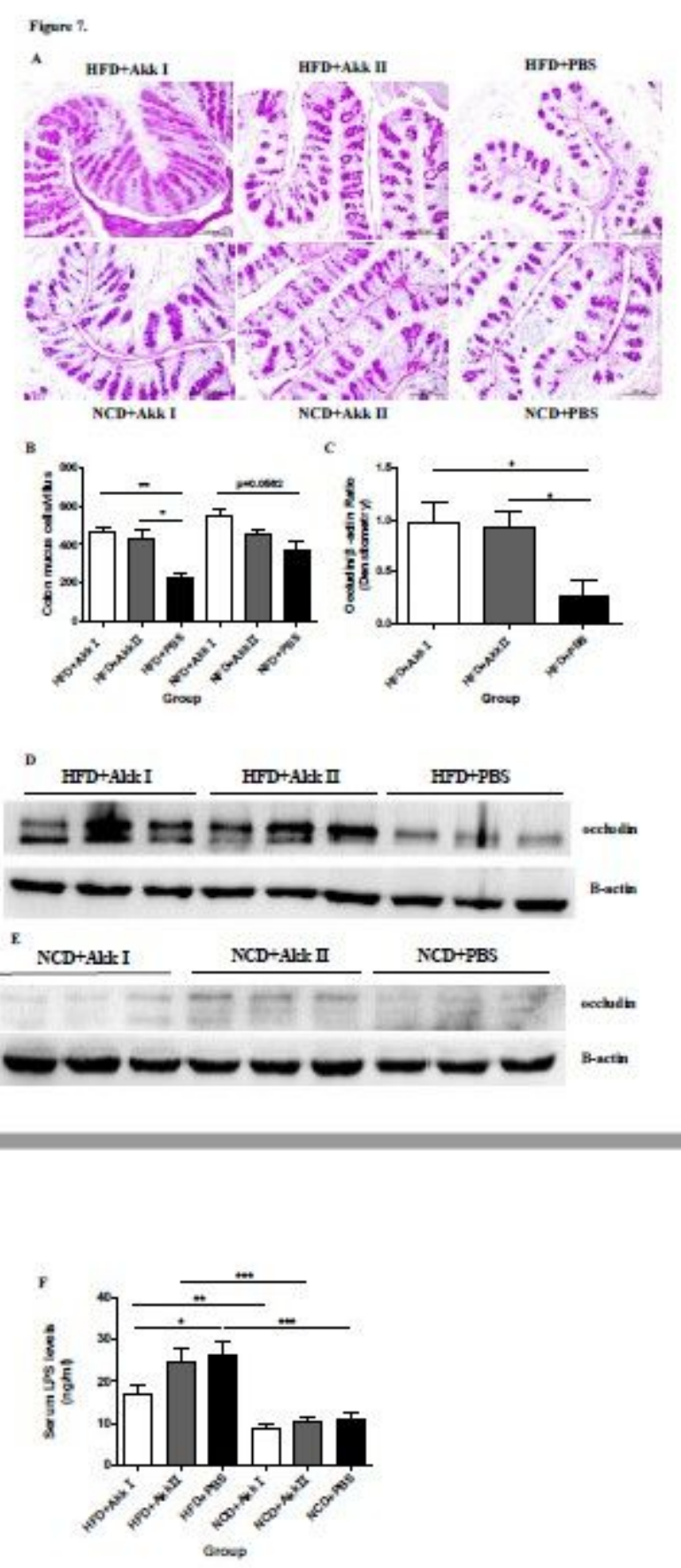

\section{Figure 7}

Akkermansia muciniphila improved endotoxemia and reversed the damage of the intestinal barrier in HFD mice. To assess the level of endotoxemia in mice, serum LPS levels were analyzed by ELISA (F). Intestinal barrier function was determined primarily by PAS staining of mucous cells and expression of the tight junction proteins in the colon. (A) Representative images of PAS staining are shown and (B) quantification of colonic mucus cells was identified by Image-Pro Plus 6.0 software. (D, E) Representative 
western blot of colon lysates in HFD groups and NCD groups. (C) The expression levels of tight junction protein in HFD groups were quantified using Image-Pro Plus 6.0 software. Data are presented as the mean \pm SEM with $n=3-8$ in each group. ${ }^{*} P<0.05 ;{ }^{* * P}<0.01 ; * \star * P<0.001$.

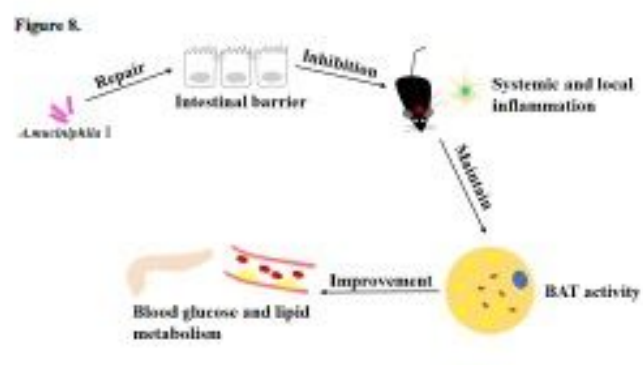

\section{Figure 8}

Possible action pathway of A.muciniphila I. A.muciniphila I treatment could repair damaged intestinal barrier induced by HFD, thus alleviating endotoxemia, inhibited systemic and local inflammation, maintained BAT activity and improved blood glucose and lipid metabolism.

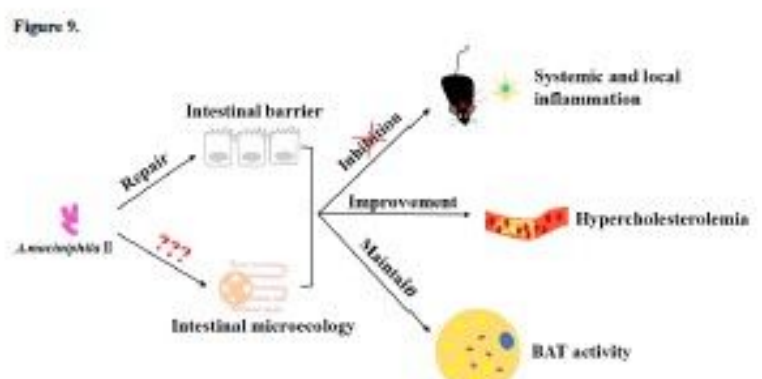

\section{Figure 9}

Possible action pathway of A.muciniphila II. A.muciniphila II treatment did not alleviate endotoxemia, and did not inhibit systemic or local inflammation. However, it could repair the intestinal barrier damage induced by HFD, which might due to its effects on intestinal microecology. Questions about the mechanism by which A.muciniphila II improved hyperlipidemia and brown fat activity remain.

\section{Supplementary Files}

This is a list of supplementary files associated with this preprint. Click to download.

- ARRIVEGuidelinesChecklist.pdf 